\title{
Long-axis rotational volvulus in an ileal J-pouch anal anastomosis: A preventable rare complication
}

\author{
Virgilio V. George*, Alyssa Fajardo \\ Department of Surgery, Indiana University School of Medicine, Indianapolis, Indiana; ${ }^{*}$ Corresponding Author: vigeorge@iupui.edu
}

Received 22 November 2013; revised 20 December 2013; accepted 7 January 2014

Copyright (C 2014 Virgilio V. George, Alyssa Fajardo. This is an open access article distributed under the Creative Commons Attribution License, which permits unrestricted use, distribution, and reproduction in any medium, provided the original work is properly cited. In accordance of the Creative Commons Attribution License all Copyrights (C) 2014 are reserved for SCIRP and the owner of the intellectual property Virgilio V. George, Alyssa Fajardo. All Copyright (C 2014 are guarded by law and by SCIRP as a guardian.

\section{ABSTRACT}

Puropose: This study was designed to report a very rare long-term complication of ileal-Jpouch anal anastomosis: An 180 degree longaxial rotational volvulus causing $\mathrm{J}$ pouch obstruction. Methods: An Ovid and Medline search using the following keywords was performed: $J$ pouch ileoanal anastomosis, J-pouch ileoanal anastomosis complications, J-pouch volvulus, J-pouch complications, restorative proctocolectomy complications, and restorative proctocolectomy volvulus. One J-pouch ileoanal anastomosis $180^{\circ}$ volvulus report was found [1]. Result: We describe a long-axis $180^{\circ}$ rotational volvulus complication of a J-pouch ileoanal anastomosis. The $\mathrm{J}$ pouch was performed three years prior after the laparoscopic total proctocolectomy for chronic ulcerative colitis. Pouch excision and new ileoanal J-pouch surgery were then performed along with pexy using alloderm mesh placement with excellent outcomes. Conclusion: Long-axis $180^{\circ}$ rotational volvulus is a rare complication of a J-pouch ileoanal anastomosis. Pouch dysfunction after a long-axis rotational volvulus is an uncommon cause of acute abdomen. Lack of adhesions and pouch size are risk factors for the pouch torsion. Prompt diagnosis and treatment are essential for the pouch salvage. Simple abdominal x-ray, barium enema and CT scan represent important tools for diagnosis. Salvage surgery should be performed even if detorsion and decompression of the affected bowel are achieved. Surgery has excellent outcomes if performed after the prompt diagnosis. Pouch pexy should be done to prevent recurrent volvulus.

\section{KEYWORDS}

Ileal Pouch; Ileal Anal Anastomosis; Malrotation

\section{INTRODUCTION}

This case report was designed to analyze a rare longterm complication of ileal J-pouch anal anastomosis performed in an ulcerative colitis patient. Ulcerative colitis is a chronic idiopathic inflammatory disease of the colorectal mucosa. This disease affects approximately 500,000 to 1,000,000 people in the United States [2-5]; 25\% - 33\% of whom require some form of surgical treatment [6]. Restorative proctocolectomy with ileal pouch anal anastomosis is the surgical treatment of a choice in patients with medically refractory disease or evidence of dysplasia. This surgical approach has evolved significantly since it was first reported [7]. The implementation of laparoscopic technique has shown better outcomes $[8,9]$. It is thought that the laparoscopic approach reduces tissue trauma, thus decreasing adhesions. However, the lack of retroperitoneal pexy increases the possibility of torsion.

A number of significant short and long-term complications have been reported: small bowel obstruction, pouch leakage, anastomotic stricture, fistulas, and pouchitis being the most common. The axial rotation of an ileal Jpouch anal anastomosis could compromise its venous return, causing edema, ischemia, necrosis, perforation and peritonitis.

This life threatening complication requires prompt diagnosis and treatment. When suspected, a simple abdominal X-ray, barium enema, and/or gastrografin enema should be used to make the diagnosis; CT scan and MRI can also be used [10-13]. When present, therapy should be started immediately. Resuscitation, nasogastric tube decompression, and detorsion with proctoscopy or flexi- 
ble sigmoidoscopy are the first line of therapy. Temporary rectal tube decompression can be considered. If this approach fails, a combined abdominal salvage surgery should be performed to release the affected bowel, prevent future devastating complications, and improve pouch function in most cases [14,15]. Pouchopexy should be considered in order to prevent future recurrences $[16,17]$.

\section{CASE REPORT}

A 34-year-old female with a history of ulcerative colitis treated three years prior with laparoscopic total proctocolectomy and ileal J-pouch anal anastomosis presented with complaints of a 24 hour loss of appetite, progressive abdominal distention, and obstipation. She reported gray rectal mucus discharge and lower back pain, low grade fever, but denied nausea or vomiting.

On physical examination she looked frail, apyrexic, and in obvious discomfort. Her vital signs were: temperature: $90.8^{\circ} \mathrm{F}$; blood pressure: 110/80 mm Hg; heart rate: 118 beats per minute; respiratory rate: 20 respirations per minute. Her abdomen was distended with diminished bowel sounds, soft but with mild central tenderness. After resuscitation with IV fluids, torsion of the pouch was diagnosed with abdominal XR (Figure 1), CT scan, rigid proctoscopy, and gastrograffin enema (Figure 2). We attempted to decompress the dilated pouch using a finger-guided Medina catheter and sigmoidoscope. This provided some relief with passage of flatus and liquid

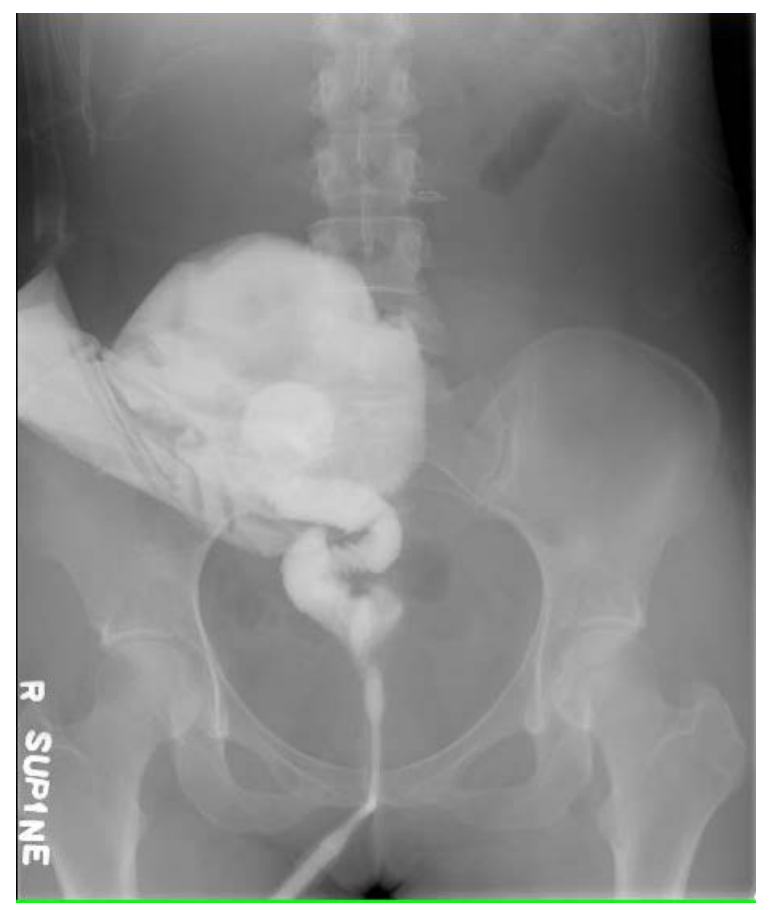

Figure 1. A gastrograffin enema demonstrates pouch distension and rotation around the EEA anastomosis.

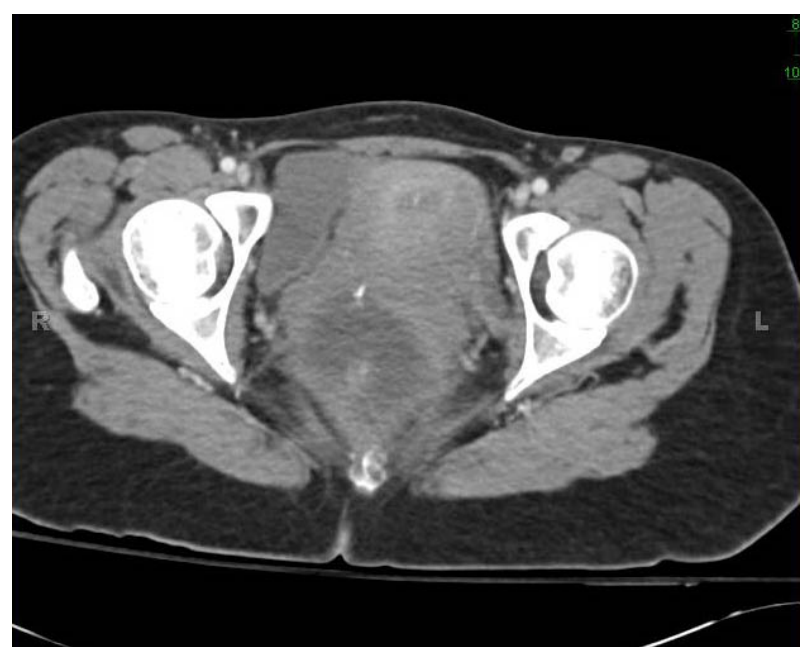

(a)

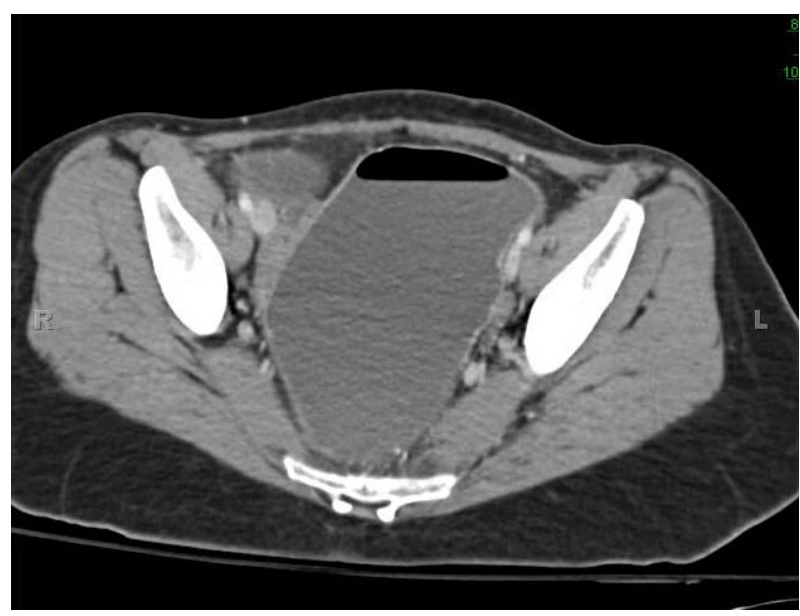

(b)

Figure 2. CT scan demonstrating pouch torsion and proximal dilation. (a) Torsion at the level of ileoanal anastomosis; (b) Dilatation of the J pouch proximal.

stool. Though the pouch demonstrated no injury or perforation, too much edema was present for proper decompression, and the patient was immediately admitted to the colorectal surgery service.

The patient was electively taken to the operative room for abdominal exploration. A massively dilated pouch was discovered without adhesions to the pelvic side wall or the mesenteric surface. The pouch was excised and a new pouch was created using the old ileostomy site as its apex. This procedure could be done without tension and a hand-sewn ileo-anal anastomosis was completed. The new pouch was secured with Alloderm sutured into the presacral space to create a pexy. A temporary loop ileostomy was created in order to protect the new pouch and a presacral drain was placed to prevent formation of collections. The post-operative period was uncomplicated. She was started on a clear liquid diet the day after surgery, which was quickly advanced as tolerated, and the 
patient was discharged home on post-operative day 5 . The presacral drain was removed and she reported 2 to 3 bowel movements despite diversion. A mild anastomotic stricture was easily treated with digital dilation during clinic follow up.

Three months later, the pouch anastomosis appeared normal in postoperative studies. She returned to the OR for a loop ileostomy takedown. During this intervention, no intra-abdominal collections were discovered and few adhesions were found. Her progression was appropriate, though she did complain of colicky abdominal pain. After her intervention, she reported 6 to 8 bowel movements a day without nocturnal leakage, and required the use of Imodium to reduce her frequent stools.

\section{DISCUSSION}

Long-axis rotational volvulus is an uncommon complication of ileal J-pouch anal anastomosis. The characteristic $180^{\circ}$ rotation of the pouch can compromise its blood supply, jeopardizing its function even if prompt diagnosis and decompression are achieved at presentation.

Loss of appetite, abdominal distention, absence of bowel movements, and the inability to pass flatus should alert the surgeon that an obstruction exists. Abdominal $\mathrm{X}$-ray, contrast enema, and sigmoidoscopy are diagnostic. Guided Medina catheter and rectal tube placement represent important tools for decompression. The utility of flexible sigmoidoscopy in the treatment of sigmoid volvulus is well known [18], but its therapeutic role in ileal J-pouch torsion is unproven.

There is no consensus about risk factors for J-pouch torsion but we believe that lack of adhesions and large pouch size could play an important role in the development of this complication. Other authors sustain that risk factors for pouch torsion are asymmetric orientation and pouch configuration [17]. The implementation of a laparoscopic approach in inflammatory bowel disease patients can decrease adhesion formation, thus increasing the risk of volvulus.

It is known that $\mathrm{W}$-pouches are more prone to torsion than J-pouches, probably because they have all the characteristics described above. This possibility may increase when the pouch is distended. The orientation, symmetry, and shortness of a J-pouch decrease the possibility of torsion and obstruction, and implementation of pouchopexy physically supports the pouch which further reduces the risk. When pouch torsion and obstruction is suspected and a conservative treatment fails to decompress the affected loop and release the obstruction, pouch excision and creation of a new pouch is one of the best surgical approaches [19] and has satisfactory outcomes $[15,20]$.

In our case, surgery was performed and pouch func- tion was assessed using markers such as number of daytime bowel movements, nocturnal leakage, and the need for drugs to control bowel activity, with expected results.

\section{CONCLUSION}

Rotational volvulus is an uncommon complication of ileal J-pouch anal anastomosis. It's a very rare cause of bowel dysfunction and bowel obstruction. The rotation of the pouch can compromise its blood supply, jeopardizing its function even if prompt diagnosis and decompression are essential.

\section{REFERENCES}

[1] Swarnkar, K. Hopper, N. Ryder, J. Feroz, A. and Stephenson, B.M. (2004) 3 years follow-up of a twisted ileoanal pouch. Colorectal Disease, 6, 133-134. http://dx.doi.org/10.1111/j.1463-1318.2004.00575.x

[2] Swarnkar, K., Hopper, N., Ryder, J., Feroz, A. and Stephenson, B. (2004) 3 years follow-up of a twisted ileoanal pouch. Colorectal Disease, 6, 133-134. http://dx.doi.org/10.1111/j.1463-1318.2004.00575.x

[3] Cohen, R., Yamashita, B. and Diamond, R. (2006) Quality of life after proctocolectomy with ileoanal anastomosis for patients with ulcerative colitis. Journal of Clinical Gastroenterology, 40, 669-677. http://dx.doi.org/10.1097/00004836-200609000-00002

[4] National Digestive Disease Information Clearinghouse (NDDIC) (2007) Digestive disease statistics. http://digestive.niddk.nih.gov/statistics/.htm/

[5] Loftus, Jr., E.V. (2004) Clinical epidemiology of inflammatory bowel disease: Incidence, prevalence and environmental influence. Gastroenterology, 126, 1504-1517.

[6] Shen, B., Fazio, V.W., Remzi, F.H. and Lashner, B.A. (2005) Clinical approach to diseases of ileal pouch-anal anastomosis. American Journal of Gastroenterology, 100, 2796-807. http://dx.doi.org/10.1111/j.1572-0241.2005.00278.x

[7] Parks, A.G. and Nicholls, R.J. (1978) Proctocolectomy without ileostomy for ulcerative colitis. British Medical Journal, 2, 85-88. http://dx.doi.org/10.1136/bmj.2.6130.85

[8] Larson, D., Dozois, E., Piotrowicz, K.R.N., Cima, R., Wolff, B. and Young-Fadok, T. (2005) Laparoscopic-assisted vs. open ileal pouch anal anastomosis: Functional outcomes in a case-matched series. Diseases of the Colon \& Rectum, 48, 1845-1850. http://dx.doi.org/10.1007/s10350-005-0143-4

[9] Maartense, S., Dunker, M., Slors, J.F.. Cuesta, M., Gouma, D., Van Deventer, S., Van Bodegraven, A. and Bemeldan, W. (2004) Hand-assisted laparoscopic versus open restorative proctocolectomy with ileal pouch anal anastomosis: A randomized trial. Annals of Surgery, 540, 984991.

[10] Crema, M.D., Richarme, D., Azizi, L., Hoeffel, C.C., Tubiana, J.M. and Arrive, L. (2006) Pouchography, CT, 
and MRI features of ileal $\mathrm{J}$ pouch-anal anastomosis. American Journal of Roentgenology, 187, W594-W603. http://dx.doi.org/10.2214/AJR.05.0870

[11] Hagen, G., Kolmannskog, F., Aasen, S., Bakka, A., Lotveit, T. and Mathisen, O. (1999) Radiology of Ileal J anal pouch anastomosis. Acta Radiologica, 64, 563-568. http://dx.doi.org/10.3109/02841859309175408

[12] Dolinsky, D., Levine, M.S., Rubesin, SE., Laufer, I. and Rombeau, J.L. (2007) Utility of contrast enema for detecting anastomotic strictures after total proctocolectomy and ileal pouch-anal anastomosis. American Journal of Roentgenology, 189, 25-29. http://dx.doi.org/10.2214/AJR.06.1382

[13] Catalano, O. (1996) Small bowel volvulus following ileal pouch-anal anastomosis: CT demonstration. European Journal of Radiology, 23, 115-117. http://dx.doi.org/10.1016/0720-048X(96)01065-0

[14] Tulchinsky, H., Cohen, CR. and Nicholls, R.J. (2003) Salvage surgery after restorative proctocolectomy. British Journal of Surgery, 90, 909-921. http://dx.doi.org/10.1002/bjs.4278

[15] Korsgen, S., Nikiteas, N., Ogunbiyi, O. and Keighley, A. (1996) Results from pouch salvage. British Journal of Surgery, 83, 372-374. http://dx.doi.org/10.1002/bjs.1800830325
[16] Reed, T., Schoetz, D., Marcello, P., Roberts, P., Coller, J., Murray, J. and Rusin, L. (1997) Afferent limb obstruction complicating ileal pouch anal anastomosis. Diseases of Colon \& Rectum, 40, 566-569.

[17] Ullah, Z., Fajobi, O. and Bhargava, A. (2007) Long-axis rotational volvulus in a W ileoanal pouch: An unusual but potentially preventable problem. Diseases of the Colon \& Rectum, 50, 540-543. http://dx.doi.org/10.1007/s10350-006-0825-6

[18] Turan, M., Sen, M., Karadayi, K., Koyuncu, A., Topcu, O., Yildirir, C. and Duman, M. (2004) Our sigmoid colon volvulus experience and benefits of colonoscope in detortion process. Revista Espanola de Enfermedades Digestivas, 96, 32-35. http://dx.doi.org/10.4321/S1130-01082004000100005

[19] Sagar, P.M., Dozois, R.R., Wolff, B.G. and Kelly, K.A. (1996) Disconnection, pouch revision and reconnection of the ileal pouch-anal anastomosis. British Journal of Surgery, 83, 1401-1405. http://dx.doi.org/10.1002/bjs.1800831025

[20] Dayton, M. (2000) Redo ileal pouch-anal anastomosis for malfunctioning pouches: Acceptable alternative to permanent ileostomy? American Journal of Surgery, 180, 561-565. 